\title{
Cosmologically viable low-energy supersymmetry breaking
}

\author{
Anson Hook ${ }^{*}$ \\ Maryland Center for Fundamental Physics, Department of Physics, \\ University of Maryland, College Park, Maryland 20742, USA \\ Robert McGehee ${ }^{\dagger}$ \\ Department of Physics, University of California, Berkeley, California 94720, USA \\ and Theoretical Physics Group, Lawrence Berkeley National Laboratory, \\ Berkeley, California 94720, USA \\ Hitoshi Murayama \\ Department of Physics, University of California, Berkeley, California 94720, USA \\ Theoretical Physics Group, Lawrence Berkeley National Laboratory, Berkeley, California 94720, USA \\ and Kavli Institute for the Physics and Mathematics of the Universe (WPI), \\ University of Tokyo Institutes for Advanced Study, University of Tokyo, Kashiwa 277-8583, Japan
}

(Received 14 September 2018; published 28 December 2018)

\begin{abstract}
A recent cosmological bound on the gravitino mass, $m_{3 / 2}<4.7 \mathrm{eV}$, together with LHC results on the Higgs mass and direct searches, excludes minimal gauge mediation with high reheating temperatures. We discuss a minimal, vector-mediated model which incorporates the seesaw mechanism for neutrino masses, allows for thermal leptogenesis, ameliorates the $\mu$ problem, and achieves the observed Higgs mass and a gravitino as light as $1-2 \mathrm{eV}$.
\end{abstract}

DOI: $10.1103 /$ PhysRevD.98.115036

\section{INTRODUCTION}

Supersymmetry (SUSY) is a well motivated theory beyond the standard model. It offers a solution to the hierarchy problem and allows for gauge unification (see, e.g., [1]). Models with SUSY breaking at low energies are especially interesting because they imply light gravitinos which can be produced at colliders, enabling experimental tests of the SUSY-breaking mechanism [2]. Gauge mediation is the most studied way to do this [3-5].

However, the window on the usual gauge mediation is quickly closing as cosmological and LHC bounds eliminate parameter space from both ends. Decreasing upper bounds on the gravitino mass from cosmological data are decreasing the upper bound on the SUSY-breaking scale, $\sqrt{F}$, because $m_{3 / 2}=F / \sqrt{3} M_{\mathrm{Pl}}$. Increasing bounds on the gaugino masses from the LHC and the $125 \mathrm{GeV}$ Higgs mass are simultaneously increasing the lower bound on $\sqrt{F}$.

\footnotetext{
*hook@umd.edu

robertmcgehee@berkeley.edu

"hitoshi@berkeley.edu; hitoshi.murayama@ipmu.jp
}

Published by the American Physical Society under the terms of the Creative Commons Attribution 4.0 International license. Further distribution of this work must maintain attribution to the author(s) and the published article's title, journal citation, and DOI. Funded by SCOAP ${ }^{3}$.
For most of the gravitino-mass range, if it is the stable lightest supersymmetric particle (LSP), it overcloses the Universe without an unnaturally low reheating temperature $[6,7]$. Such a low reheating temperature makes baryogenesis difficult as well. In particular, thermal leptogenesis requires $T_{R}>10^{9} \mathrm{GeV}$ [8]. A very light gravitino, $m_{3 / 2}<0.24 \mathrm{keV}$, does not overclose the Universe even when it is thermalized. But it constitutes a hot (or warm) dark matter component and suppresses the structure of the Universe at small scales. According to a very recent study, cosmic microwave background (CMB) lensing and cosmic shear constrain its mass to be $m_{3 / 2}<4.7 \mathrm{eV}$ [9]. Figure 1 summarizes these cosmological bounds on $m_{3 / 2}$, as well as others, and demonstrates the shrinking parameter space for any models with gravitinos lighter than roughly $10^{5} \mathrm{GeV}$.

In minimal, gauge-mediated models, on the other hand, the $125 \mathrm{GeV}$ Higgs mass requires a large stop mass. This implies $\sqrt{F} \gtrsim 10^{3} \mathrm{TeV}$. Hence, the gravitino is heavier than $360 \mathrm{eV}$ for one messenger or $60 \mathrm{eV}$ for five messengers, which is the maximum number allowed by perturbative gauge unification [13]. Therefore, minimal, gauge-mediated models are excluded if the reheating temperature is high.

This gravitino problem is often ignored in literature which attempts to achieve the $125 \mathrm{GeV}$ Higgs mass via gauge mediation, e.g., using $A$ terms from renormalization group equation flow [14] or nondecoupling $D$ terms $[15,16]$. 


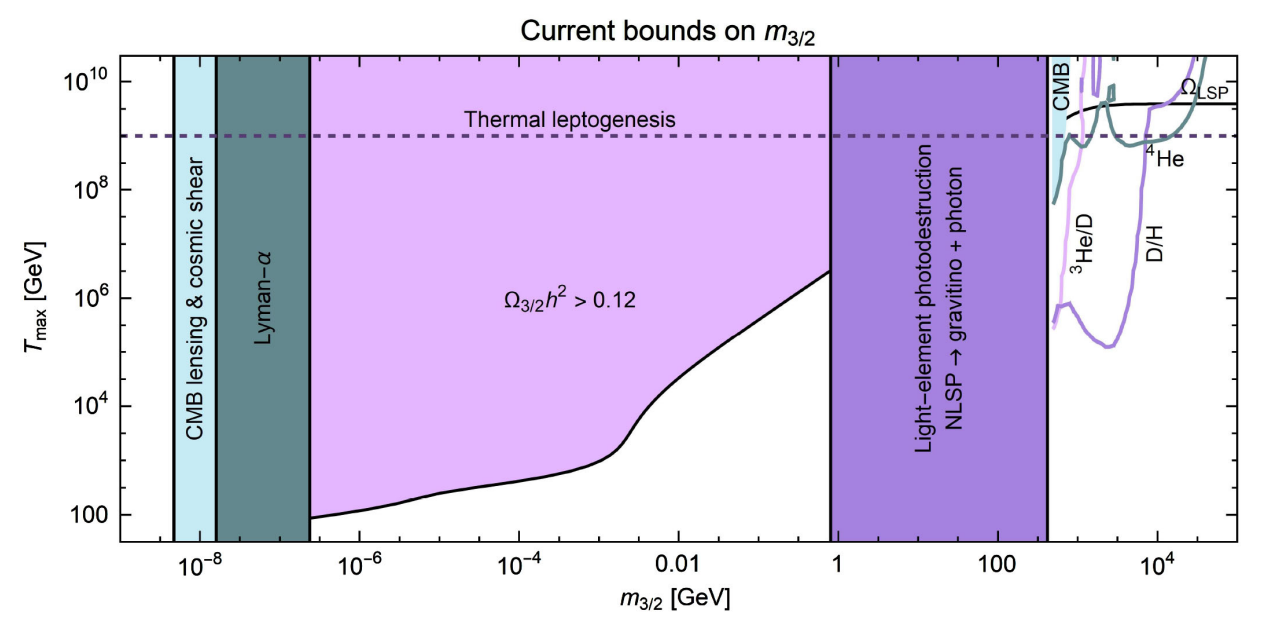

FIG. 1. A compilation of bounds in the $m_{3 / 2}-T_{\max }$ plane, where $T_{\max }$ is the maximum temperature from which the usual, radiationdominated universe starts. In inflationary models, $T_{\max }$ corresponds to the reheat temperature, $T_{R}$. The bound from CMB lensing and cosmic shear is in light blue [9]. The Lyman- $\alpha$ bound from Ref. [10] is in dark green. The overclosure bound for gravitinos heavier than $\gtrsim 0.2 \mathrm{keV}$ is in light purple [6,10]. We recalculated this limit with current measurements of the Hubble scale factor and dark matter abundance assuming $M_{1}=417 \mathrm{GeV}$ and the ratio $M_{1}: M_{2}: M_{3}=1: 2: 7$. The bound from light-element photodestruction is in dark purple [11]. The bounds from a long-lived gravitino affecting light-element abundances after Big Bang nucleosynthesis are taken from Fig. 15 of Ref. [12] for $m_{\mathrm{LSP}}=M_{1}=417 \mathrm{GeV}$. The lower bound on the reheat temperature from thermal leptogenesis is the darkpurple dashed line [8].

A nonminimal Higgs sector or strongly coupled messengers may achieve $m_{3 / 2} \lesssim 16 \mathrm{eV}$ [17], but it is unclear if the gravitino mass can be pushed below $4.7 \mathrm{eV}$. These problems can be ameliorated by using the next-to-minimal supersymmetric Standard Model (NMSSM) or Dirac-NMSSM [18], coupling the Higgs to messengers [19], or having a strongly coupled messenger sector [20-23]. However, these models are quite intricate.

In this paper, we elaborate on a simple, vector-mediated model proposed by Hook and Murayama which breaks SUSY at tree level with a $U(1) D$ term and evades all gravitino bounds [24]. We discuss electroweak symmetry breaking (EWSB) in detail to obtain the correct Higgs mass while avoiding all LHC bounds on minimal supersymmetric Standard Model (MSSM) particles. We also introduce a new $U(1)$ charge assignment where the right-handed neutrino is neutral to allow the seesaw mechanism for neutrino masses and thermal leptogenesis [25]. Additionally, this model produces a split spectrum usually found in gravity-mediated models [26].

We first review the basics of SUSY breaking via vector mediation. ${ }^{1}$ If we want a cosmologically viable, light gravitino and a naturally high reheating temperature, Fig. 1 clearly shows it must be very light. ${ }^{2}$ A first attempt is to use a low-energy, gauge-mediated model. However, normal, gauge-mediated models have gaugino and scalar

\footnotetext{
${ }^{1}$ Not to be confused with the identically named theory in Ref. [27] which has a different mechanism.

${ }^{2}$ An alternative way to achieve a high reheating temperature is anomaly mediation [28,29].
}

masses at loop level. Then the low, SUSY-breaking scale results in spartners too light for the current LHC bounds. Thus, we want a low-energy, gauge-mediated model with scalar masses at tree level.

However, there is a "no-go theorem" against models which break SUSY at tree level [30-32]. The problem is the tree-level identity $\operatorname{STr}\left(\mathcal{M}^{2}\right)=0$ which usually implies SUSY breaking cannot occur at the tree level. This is because anomaly cancellation requires both positive and negative $U(1)$ charges which gives some scalars negative soft masses at tree level from a $U(1) D$ term.

In vector mediation, there are vectorlike messenger fields. ${ }^{3}$ We assign positive $U(1)$ charges to all sfermions and negative charges to all vectorlike messengers. Their vectorlike masses overcome their negative soft masses so that no scalars are tachyonic. Thus, vector mediation allows tree-level scalar masses and a lower SUSY-breaking scale. This gives a lighter gravitino while getting the Higgs mass correct and avoiding current bounds on MSSM particles. The lower scale also ameliorates the so-called $\mu$ problem.

\section{THE MODELS}

The vector-mediated models [24] employ an $E_{6}$-inspired particle content that consists of three families of the fundamental representation decomposed into $S O(10) \times$ $U(1)_{\psi}$ as

\footnotetext{
${ }^{3}$ References [33,34] also used a $U(1) D$ term to mediate SUSY breaking at tree level, but at the grand unified theory scale. This resulted in a very weakly coupled gravitino and thus, very different collider and cosmology signatures [35].
} 


$$
\mathbf{2 7}=\Psi(\mathbf{1 6},+1) \oplus \Phi(\mathbf{1 0},-2) \oplus S(\mathbf{1},+4),
$$

where $\Psi$ contains the SM fermions and $\Phi$ contains two families of messengers and the MSSM Higgs doublets with their color triplets. Because the messengers and Higgs color triplets have different couplings than the Higgs doublets, we distinguish between generations using subscripts with $\Phi_{1} \equiv\left(T_{u}, H_{u}\right)+\left(T_{d}, H_{d}\right)$ where $T$ are the color triplets. Since these Higgs color triplets' masses are not at the grand-unified-theory scale, they prevent gauge unification. However, we can add two new electroweak doublets, uncharged under our $U(1)$, with the same mass as the Higgs color triplets in order to form complete $S U(5)$ multiplets and restore gauge unification. We also include a neutral particle $Z(0)$ and a vectorlike multiplet charged under $U(1)_{\psi}, X(-4)$, and $Y(+4)$. These particles are responsible for SUSY breaking.

The superpotential for our model is

$$
\begin{aligned}
W= & M S X+\lambda Z\left(X Y-v_{S}^{2}\right) \\
& -2 T_{u} T_{d}\left(\frac{g}{2} Y+\frac{k}{2} S\right)-2 H_{u} H_{d}\left(\frac{g_{H}}{2} Y+\frac{k_{H}}{2} S\right) \\
& -\sum_{a=2}^{3} \Phi_{a} \cdot \Phi_{a}\left(\frac{g}{2} Y+\frac{k}{2} S\right) \\
& +\frac{\lambda^{S}}{2} \sum_{a=2}^{3} \Phi_{a} \cdot \Phi_{a} \sum_{b=1}^{2} S_{b}+\kappa X \sum_{a=1}^{2} N_{a} S_{a} .
\end{aligned}
$$

The three generations of $S$ are denoted $S_{1}, S_{2}$, and $S$. The first line in Eq. (2) breaks SUSY with $F$ terms and a positive $D$ term. It can be dynamically realized as the low-energy effective theory of an Izawa-YanagidaIntriligator- Thomas (IYIT) model with $4 S U(2)$ doublets with appropriate $U(1)$ charges [36,37]. The second and third lines are standard messenger interactions and generate the usual, gauge-mediated gaugino masses at one loop. We have allowed the MSSM Higgs doublets, $H_{u}$ and $H_{d}$, to have different couplings to $Y$ and $S$ than their color triplets, $T_{u}$ and $T_{d}$, in order to satisfy EWSB conditions. Each generation of $\Phi$ could have different couplings to $Y$ and $S$, but we set them equal to maximize the gaugino masses (see Sec. III). The fourth line gives the $S_{1,2}$ fermion masses by introducing two neutral fields $N_{1,2}$, while the $S_{1,2}$ scalars also acquire a mass from the $D$ term. We have the interaction $W \supset S_{1,2} \Phi \Phi$ in line four to allow $S_{1,2}$ and $N_{1,2}$ to decay so that they do not overclose the universe.

In this paper, we consider two different charge assignments under a new $U(1)$ : the original-charge assignment corresponding to $U(1)_{\psi}$ and a new, seesaw-charge assignment corresponding to a different $U(1)_{S S}$. For clarity, we distinguish between these charge assignments using these subscripts and omit a subscript when talking about either charge assignment generally. We explore the viability of models with each of these charge assignments separately.

The right-handed neutrino has charge +1 under $U(1)_{\psi}$, as do all SM fermions by virtue of Eq. (1). Thus, gauging $U(1)_{\psi}$ does not allow the seesaw mechanism to generate neutrino masses. However, the right-handed neutrino can be made neutral by instead gauging a linear combination of $U(1)_{\psi}$ and $U(1)_{\chi}$, where a given $S O(10)$ representation is decomposed into $S U(5) \times U(1)_{\chi}$. The linear combination we gauge is

$$
Q_{S S}=\frac{1}{4}\left(5 Q_{\psi}+Q_{\chi}\right)
$$

Using this charge, we find the decomposition of $\Psi(\mathbf{1 6},+1)$ into $S U(5) \times U(1)_{S S}$ is

$$
\mathbf{1 6}=\mathbf{1 0}(+1) \oplus \overline{\mathbf{5}}(+2) \oplus \mathbf{1}(0) .
$$

The right-handed neutrino of each family of $\Psi$ is neutral under $U(1)_{S S}$ by virtue of Eq. (4), allowing the seesaw mechanism for neutrino masses. The decomposition of $\Phi(\mathbf{1 0},-2)$ into $S U(5) \times U(1)_{S S}$ is

$$
\mathbf{1 0}=\mathbf{5}(-2) \oplus \overline{\mathbf{5}}(-3)
$$

Additionally, each $S$ has charge $Q_{S S}=5, X\left(Q_{S S}=-5\right)$, $Y\left(Q_{S S}=+5\right)$, and $Z$ remains neutral.

We do not have the usual $\mu$-term as it is not gauge invariant under $U(1)_{\psi}$ or $U(1)_{S S}$. Similar to the NMSSM, it is generated by the expectation values of $Y$ and $S$. For the two charge assignments, we have to introduce different interactions and discrete symmetries to allow the color triplets to decay while preventing proton decay.

For the seesaw-charge assignment, we introduce the interaction $W \supset \frac{1}{M_{\mathrm{p}}} S Q T_{d} H_{d}$ to allow for the color triplets in $\Phi$ to decay. We assign negative matter parity to all SM multiplets in $\Psi$ and to the color triplets in $\Phi$, but positive matter parity to the electroweak doublets in $\Phi$ to prevent proton decay. ${ }^{4}$ This is consistent with assigning $B=+1 / 3$ to $T_{u}$ and $B=-1 / 3$ to $T_{d}$.

For the original-charge assignment, we introduce the interaction $W \supset T_{d} \bar{u} \bar{d}$ to allow for the color triplets in $\Phi$ to decay. We enforce lepton-number conservation to prevent proton decay. Since the right-handed neutrinos are charged under $U(1)_{\psi}$, this requirement is consistent with the neutrinos having Dirac masses.

For the seesaw-charge assignment in Eq. (5), the MSSM Higgs soft masses satisfy $m_{H_{u}}^{2}>m_{H_{d}}^{2}$ at tree level since the $D$ term is positive and $-2>-3$. This is problematic for EWSB. To help, we introduce kinetic mixing between our

${ }^{4}$ R. M. thanks T. Yanagida for pointing out this issue. Matterparity assignment is related to $R$-parity assignment via $P_{M}=$ $P_{R} \times(-1)^{2 s}$, where $s$ is the spin of the particle in question. 
$U(1)_{S S}$ [or $U(1)_{\psi}$ for the original-charge assignment] and $U(1)_{Y}$. Following Ref. [38], this kinetic mixing can be written as a cross term between the $D$ terms associated with the two $U(1)$ 's as

$$
V \supset g^{\prime} \chi e \sum_{i, j} Q_{Y}^{i} Q^{j}\left|\phi_{i}\right|^{2}\left|\phi_{j}\right|^{2},
$$

where $Q_{Y}^{i}$ is the $U(1)_{Y}$ charge of the scalar $\phi_{i}, Q^{j}$ is the $U(1)$ charge, and $e$ is the $U(1)$ electric charge. The dimensionless coupling $\chi$ is a measure of the mixing, and we have dropped terms proportional to $\chi^{2} \cdot \chi$ is naturally small and is generated by one-loop diagrams with particles charged under both $U(1)$ 's [38]. Note that the addition of this mixing does not change the locations of the false and true vacua. The $D$ term gives every scalar $\phi_{i}$ a mass contribution from Eq. (6),

$$
\delta m_{i}^{2}=Q_{Y}^{i} g^{\prime} \chi\langle D\rangle .
$$

Since $H_{u}$ and $H_{d}$ have opposite $U(1)_{Y}$ charges, Eq. (7) aids EWSB in models with the seesaw-charge assignment.

\section{CONSTRAINTS PRIOR TO EWSB}

We explore the parameter space $\left(e, M, \lambda, v_{S}, \chi, k\right.$, $\left.g, k_{H}, g_{H}, \tan \beta\right)$ for both charge assignments to find viable models with light gravitinos which avoid the cosmological bound $m_{3 / 2}<4.7 \mathrm{eV}$. Before we verify EWSB and the Higgs mass, we must impose some constraints on our parameter space. The first suppresses tunneling to the true vacuum.

The interactions in lines two and three of Eq. (2) which give rise to the gaugino masses at one loop also generate a supersymmetric vacuum. This true vacuum appears at

$X_{0}=\frac{v_{s}^{2}}{Y_{0}}=\frac{k \Phi_{0}^{2}}{2 M}, \quad Z_{0}=\frac{g M}{k \lambda}, \quad S_{0}=-\frac{2 g M v_{S}^{2}}{k^{2} \Phi_{0}^{2}}$,

where $\Phi_{0}$ is set by requiring the $D$ term to vanish. We require the parameter space we consider to disallow substantial vacuum or thermal tunneling between the false and true vacua.

To calculate the vacuum tunneling rate, we calculate the bounce profile $\bar{\phi}(r)$ which solves

$$
\frac{d^{2} \bar{\phi}_{i}}{d r^{2}}+\frac{3}{r} \frac{d \bar{\phi}_{i}}{d r}=\frac{d V}{d \bar{\phi}_{i}}
$$

with the boundary conditions

$$
\frac{d \bar{\phi}_{i}}{d r}(r=0)=0, \quad \bar{\phi}_{i}(r \rightarrow \infty)=\phi_{0},
$$

where $\phi_{0}=\left(X_{0}, Y_{0}, 0,0,0\right)$ is the false vacuum. The spherically symmetric, four-dimensional (4D) Euclidean action of a profile $\phi(r)$ is
$S_{E}(\phi(r))=2 \pi^{2} \int_{0}^{\infty} d r r^{3}\left[\sum_{i} \frac{1}{2}\left(\frac{d \phi_{i}}{d r}\right)^{2}+V(\phi(r))\right]$,

where the sum is over all field dimensions $(X, Y, Z, S, \Phi)$. The tunneling rate per unit volume is exponentially sensitive to the bounce action $B=S_{E}(\bar{\phi})-S_{E}\left(\phi_{0}\right)$ as $\Gamma \propto$ $\exp (-B)$ [39].

The thermal bounce action, $B_{\mathrm{th}}=S_{\mathrm{th}}(\bar{\phi})-S_{\mathrm{th}}\left(\phi_{0}\right)$, is similar: the action is given by the three-dimensional (3D) version of (11) and the bounce profile solves the 3D versions of (9) and (10). The thermal tunneling rate is $\Gamma_{\text {th }} \propto T^{4} \exp \left(-B_{\text {th }} / T_{C}\right)$, where $T_{C}$ is the critical temperature at which the SUSY-invariant vacuum becomes the true minimum.

When approximating both the vacuum and thermal bounce actions, we calculate the tunneling along the straight line between the true and false vacua. The 4D vacuum and $3 \mathrm{D}$ thermal bounce actions are calculated semianalytically using results from Ref. [40]. In order for these tunneling rates to be sufficiently small, we conservatively require that $B>450$ and $B_{\mathrm{th}} / T_{c}>250$. We use the high-temperature approximation for the thermal effective potential to calculate $T_{C}$. We also assume $B_{\text {th }}$ is approximately constant and calculate it using the zero-temperature potential. For much of our parameter space, $T_{C} \lesssim v_{S}$ where this approximation breaks down. However, we find that $B_{\text {th }}$ is so large in our region of interest that this does not matter. We verify that $\operatorname{STr}\left(\mathcal{M}^{2}\right)=0$ along the straight line between the true and false vacua. We set $\kappa=0.1$ for concreteness.

To illustrate that the vacuum and thermal tunneling rates are highly suppressed, we have chosen the representative values $(M=2, \lambda=4 \pi, \chi=0, k=4 \pi)$ and set $g$ as small as possible before the lightest messengers go tachyonic [see Eq. (12) below]. See Figs. 2 and 3.

Both vacuum and thermal bounce actions are approximately independent of $\chi$ over the range of values we consider later. While $\chi=0$ is not viable for the seesaw-charge

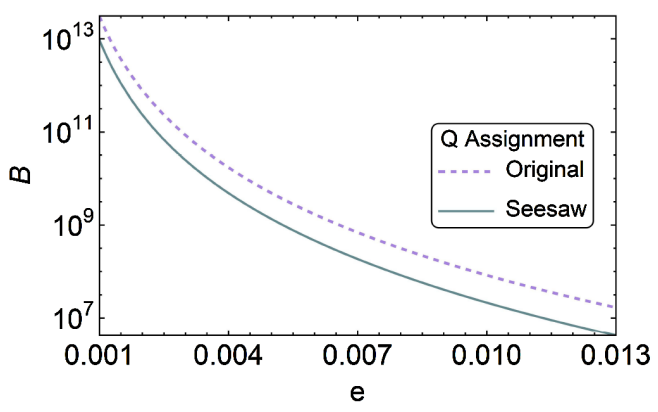

FIG. 2. Vacuum bounce action for both charge assignments. We take the representative values $(M=2, \lambda=4 \pi, \chi=0, k=4 \pi)$ and set $g$ as small as possible before the lightest messengers go tachyonic. We easily satisfy $B>450$ for the Universe to be stable. 


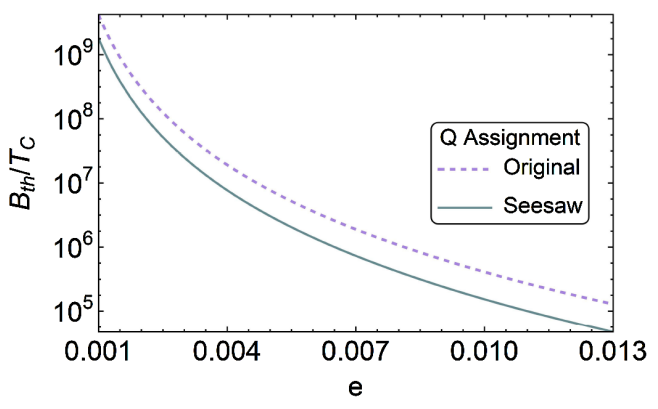

FIG. 3. $B_{\mathrm{th}} / T_{C}$ for both charge assignments. We take the same values as in Fig. 2. We easily satisfy $B_{\mathrm{th}} / T_{C}>250$ for the Universe to be stable.

assignment due to EWSB failure, these figures do not change for realistic values of $\chi$. We allow different values of $(M, \lambda, \chi)$ when conducting a full parameter-space search. However, we always take $k=4 \pi$ and minimize $g$ in order to maximize the gaugino masses (see Sec. III).

Both actions are dramatically larger than in Ref. [24] because we are considering much smaller values of $e$. One can guess that a smaller value of $e$ makes the bounce actions much larger because when $e \rightarrow \infty$, $\Phi$ is tachyonic and there is no barrier between the false and true vacua. As there is a barrier for small $e$, by continuity, one expects that smaller $e$ gives larger barriers. In the limit $e \rightarrow 0$, we numerically find that the barrier height between the two vacua increases. This is the thin-wall limit and the bounce actions increase dramatically $[39,41,42]$.

The scalar potential along the straight-line, tunneling path is quartic in one scalar field. This potential is characterized by a single, dimensionless parameter $\delta$ which can take values between 0 and 2 [40]. The thin-wall limit corresponds to the limit $\delta \rightarrow 2$. In this limit, the analytic, vacuum bounce action is proportional to $(2-\delta)^{-3}$ and the analytic, thermal bounce action is proportional to $(2-\delta)^{-2}$. For the parameters taken in Ref. [24], $\delta=1.6$. We consider points in parameter space which are much closer to the thinwall limit with $\delta \gtrsim 1.996$.

The gaugino masses are generated from one-loop diagrams with the messengers in the three families of $\Phi$. All fermions in $\Phi$ have the same mass $M_{\Phi}=g Y_{0}$. Taking into account the kinetic mixing in Eq. (7), the boson components all have the mass matrix

$$
\left(\begin{array}{cc}
M_{\Phi}^{2}+Q_{H_{u}} e D+Q_{H_{u}}^{Y} g^{\prime} \chi D & k F_{S} \\
k F_{S} & M_{\Phi}^{2}+Q_{H_{d}} e D-Q_{H_{u}}^{Y} g^{\prime} \chi D
\end{array}\right),
$$

where $Q_{H_{u}}$ is the $U(1)$ charge of fields in the $\mathbf{5}$ of $\Phi, Q_{H_{d}}$ is the charge of fields in the $\overline{\mathbf{5}}$ of $\Phi, F_{S}=M X_{0}$, and
$D=e Q_{X}\left(X_{0}^{2}-Y_{0}^{2}\right)>0 . Q_{X}=-Q_{Y}$ since $X$ and $Y$ form a vectorlike multiplet. For the bosons in the color triplets, this is the correct mass matrix under the appropriate replacement $Q_{H_{u}}^{Y}=+\frac{1}{2} \rightarrow Q_{d}^{Y}=-\frac{1}{3}$. We leave the $U(1)$ charges unspecified since we find viable models for both charge assignments. The MSSM Higgses have the same mass matrix under the replacement $k \rightarrow k_{H}, g \rightarrow g_{H}$ [see Eq. (2)].

We calculate the one-loop gaugino masses using Eq. (2.3) in Ref. [43]. All three families' color triplets and electroweak doublets contribute to the gaugino masses. The on-shell gluino mass enhancement from (s)top loops is calculated using SOFTSUSY (discussed below) [44-47].

The gaugino masses are maximized when there is a large mass hierarchy in Eq. (12). We ensure a large hierarchy by choosing parameters where the lighter scalar is light $(\approx 1 \mathrm{TeV})$. The gaugino masses are also proportional to the fermion masses so we prefer larger $g$ and larger $Y_{0}$. In order to increase the gaugino masses in units of $v_{S}$, we thus set $k=4 \pi$ and choose $g$ such that the lighter scalar in Eq. (12) is near $1 \mathrm{TeV}$. These are the prescriptions we used in Figs. 2 and 3 and allow smaller $v_{S}$.

We set $v_{S}$ by satisfying all current, gaugino-mass bounds. The ATLAS bound on the gluino mass is roughly $2.03 \mathrm{TeV}$ [48], while the CMS bound is $1.95 \mathrm{TeV}$ [49]. The ATLAS bound on the Wino mass is roughly $620 \mathrm{GeV}$, unless the Bino is heavier than $350 \mathrm{GeV}$, in which case it is $720 \mathrm{GeV}$ [50]. The Wino bound is the most stringent from direct searches. So, we use it to estimate $v_{S}$ before EWSB. But we find getting the correct Higgs mass is generally harder and therefore, determines $v_{S}$ after EWSB (see below).

The gravitino mass is given by

$$
m_{3 / 2}^{2}=\frac{V}{3 M_{\mathrm{Pl}}^{2}},
$$

where $M_{\mathrm{Pl}}=2.4 \times 10^{18} \mathrm{GeV}$. For a viable model, we require $m_{3 / 2}<4.7 \mathrm{eV}$ [9].

We always set $\chi$ negative to make $m_{H_{u}}^{2}$ lighter and help EWSB [see Eq. (12)]. Since $\tilde{\tilde{e}}$ has the greatest $U(1)_{Y}$ charge, $Q_{\bar{e}}=+1$, we determine how negative $\chi$ can be by requiring that $\tilde{\tilde{e}}$ is not tachyonic.

We also require that the massive $U(1)$ boson does not mix too much with $W$ and $Z$ to affect the $\rho$ parameter. ${ }^{5}$ We conservatively require that $\delta \rho<10^{-3}$ which imposes that $\left(\frac{e v}{M_{V}}\right)^{2}<10^{-3}$, where $v$ is the Higgs vacuum expectation value and $M_{V}$ is the mass of our $U(1)$ boson [51].

\section{CONSTRAINTS AFTER EWSB}

We use SOFTSUSY to calculate radiative EWSB. Our inputs into SOFTSUSY are the gaugino masses, trilinear

\footnotetext{
${ }^{5}$ R. M. thanks Simon Knapen on this point.
} 
couplings, sfermion masses, $m_{H_{u}}^{2}, m_{H_{d}}^{2}$, and $\tan \beta$. We can read off the MSSM Higgs parameters from Eq. (12):

$$
\begin{aligned}
\mu=g_{H} Y_{0}, & m_{H_{u}}^{2}=Q_{H_{u}} e D+\frac{1}{2} g^{\prime} \chi D, \\
B \mu=k_{H} F_{S}, & m_{H_{d}}^{2}=Q_{H_{d}} e D-\frac{1}{2} g^{\prime} \chi D .
\end{aligned}
$$

The soft, SUSY-breaking $A$ terms are all 0 in our models at tree level. SOFTSUSY effectively sets $\mu$ and $B \mu$ as output boundary conditions and therefore, $k_{H}$ and $g_{H}$ in our models.

We only input the above boundary conditions at tree level. We input the gaugino masses at one-loop level, but do not include QCD enhancements to $M_{3}$ or other, higherorder corrections. This is slightly inconsistent as we run SOFTSUSY with the 3-loop renormalization group equations and calculate the lightest MSSM Higgs both at 2-loop and 3-loop levels. When calculating the Higgs at 3-loop, SOFTSUSY also defaults to adding 2-loop Yukawa and $g_{3}$ threshold corrections to $m_{t}$ and $m_{b}$.

EWSB itself is a strong constraint. If a point in parameter space has successful radiative EWSB, there are still many additional constraints we check before considering it viable. In order to approximate EWSB using SOFTSUSY, the correction to the MSSM, Higgs-quartic coupling, $\lambda_{h}=m_{h}^{2} / v^{2}$, must be sufficiently small. We require our Higgs mass to be accurate to $0.5 \mathrm{GeV}$. This places an upper bound at roughly

$$
\delta \lambda_{h}=g_{H}^{2}+\frac{1}{2} Q_{H_{d}}^{2} e^{2}+\frac{1}{2} e g^{\prime} \chi<0.002 .
$$

This is a conservative limit because the quartic couplings due to the $F$ term and the $D$ term of heavy fields decouple and the effect is in general smaller. We do not bother including a term proportional to $k_{H}^{2}$ since $k_{H}^{2} \ll g_{H}^{2}$ generically. Equation (15) is also conservative because the $g_{H}^{2}$ piece vanishes in the limit of large or small $\tan \beta$. Due to the upper bound on $\delta \lambda_{h}$, Eq. (15) lets us only consider $e<0.021$. We find viable models only occur for smaller values of $e$. This is a nontrivial check because $\left(k_{H}, g_{H}\right)$ are set by the output boundary conditions from SOFTSUSY.

Requiring the Higgs mass to be $125 \mathrm{GeV}$ is the strongest constraint and sets the overall scale $v_{S}$ in our models. If $v_{S}$ is set to satisfy the gaugino constraints, the stop masses are too light to lift the Higgs mass. When searching for viable models, we estimate $v_{S}$ by satisfying the Wino constraint and then explore parameter space by increasing this $v_{S}$ by some factor between $\sim 2$ and 3 .

We require that the output top Yukawa is still perturbative. We calculate $y_{t}$ using Eq. (62) in Ref. [52] using the top mass in Ref. [53]. We require that $y_{t}<0.94$. We also require that the other neutral scalar Higgses are not too light. As a conservative constraint, we require the mass of the heavier $C P$-even neutral scalar, $H^{0}$, to be larger than $580 \mathrm{GeV}$ [54]. We require the mass of the $C P$-odd neutral scalar, $A^{0}$, to avoid the $\tan \beta$-dependent, ATLAS bounds in Fig. 10(b) of Ref. [55].

Since SOFTSUSY includes loop corrections to the gluino, we make sure the output gluino is still heavier than $2.03 \mathrm{TeV}$. SOFTSUSY also gives the spectrum of the neutralinos and charginos. We make sure to avoid the bound on $\tilde{\chi}_{1}^{ \pm}$and $\tilde{\chi}_{2}^{0}$ as a function of $\tilde{\chi}_{1}^{0}$ given in Ref. [50].

\section{SUSY SPECTRA AND BEST MODELS}

Figure 4 shows our search through parameter space for viable models with the seesaw-charge assignment, $Q_{S S}$, and the original-charge assignment, $Q_{\psi}$. The points correspond to viable points in the parameter space $\left(e, M, \lambda, v_{S}, \chi, k\right.$, $\left.g, k_{H}, g_{H}, \tan \beta\right)$ for which all of the constraints are satisfied. We show viable points when we calculate the Higgs mass at both the 2-loop and 3-loop levels in SOFTSUSY. The 3-loop calculation generally yields a lighter Higgs which requires a greater $v_{S}$ to obtain $125 \mathrm{GeV}$. This yields a heavier gravitino and explains the separation of viable points between the 2-loop and 3-loop calculations. We find $v_{S} \in[55,240] \mathrm{TeV}$ for the seesaw-charge assignment and $v_{S} \in[44,240] \mathrm{TeV}$ for the original-charge assignment.
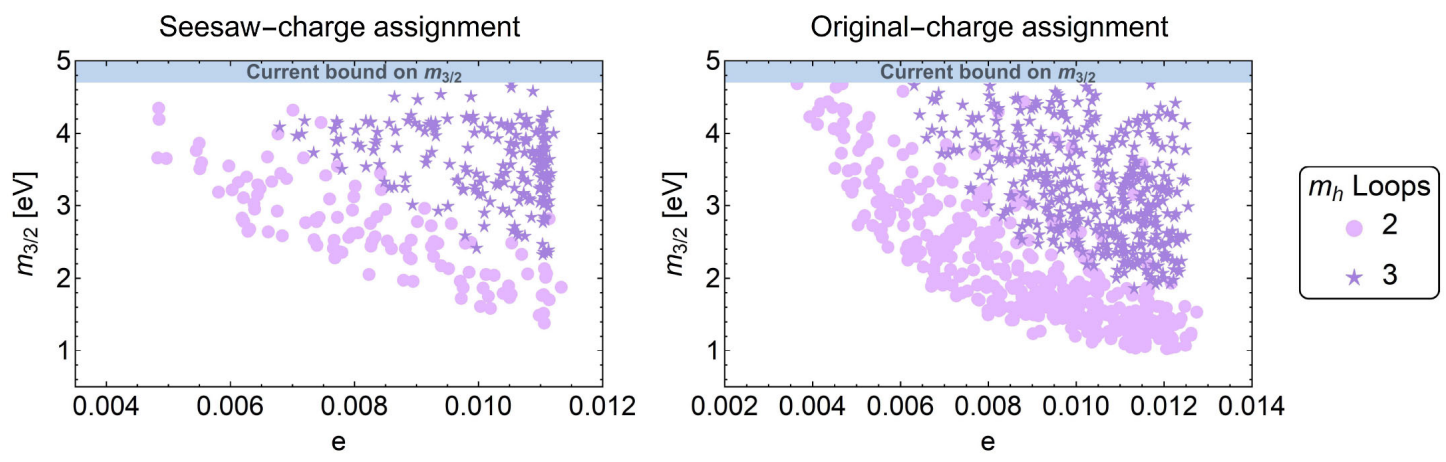

FIG. 4. $m_{3 / 2}$ vs $e$ for viable models with the seesaw-charge and original-charge assignments which satisfy all aforementioned constraints. Viable points are shown when the Higgs is calculated at both the 2-loop and 3-loop levels in SOFTSUSY. 

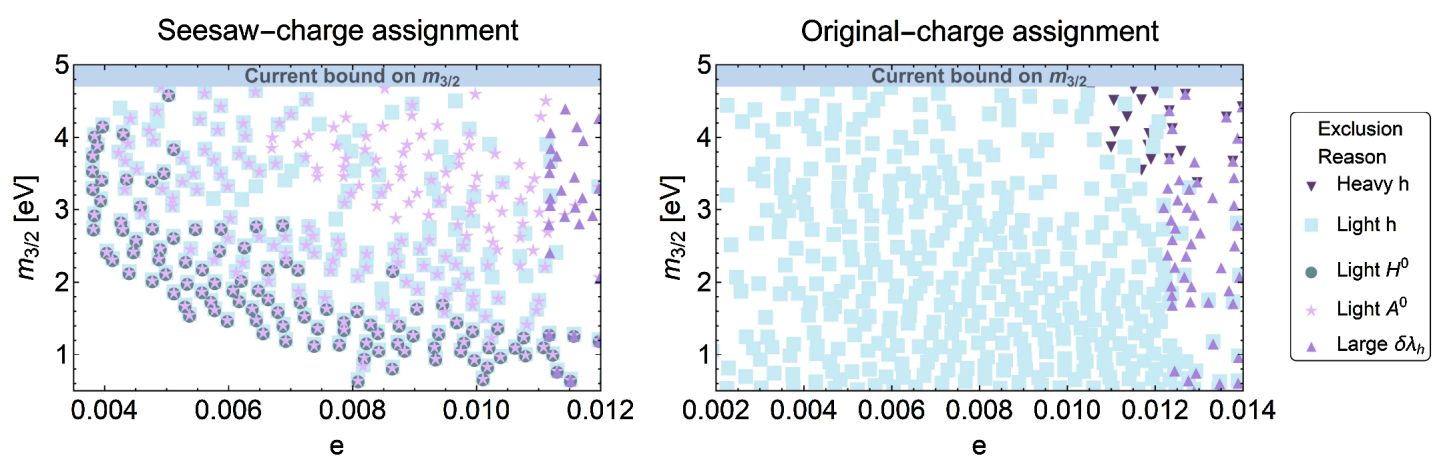

FIG. 5. $m_{3 / 2}$ vs $e$ for models with the seesaw-charge and original assignments which are excluded by the aforementioned constraints. Excluded points are shown along with their reason for exclusion when the Higgs is calculated at the 3-loop level in SOFTSUSY.

For the seesaw-charge assignment, $\chi$ is always close to its maximum value to help alleviate $m_{H_{u}}^{2}>m_{H_{d}}^{2}$, namely $\chi \in[-0.033,-0.014]$. This is naturally small and generated by one-loop diagrams of particles charged under both $U(1)$ 's [38]. However, it is not possible to take $\chi$ large enough to make $m_{H_{u}}^{2} \leq m_{H_{d}}^{2}$ without making $\tilde{\bar{e}}$ tachyonic. EWSB works due to negative, one-loop corrections to $m_{H_{u}}^{2}$ from (s)tops at the geometric mean of the stop masses. In particular, the one-loop corrections to $m_{H_{u}}^{2}$ from the (s)tops are
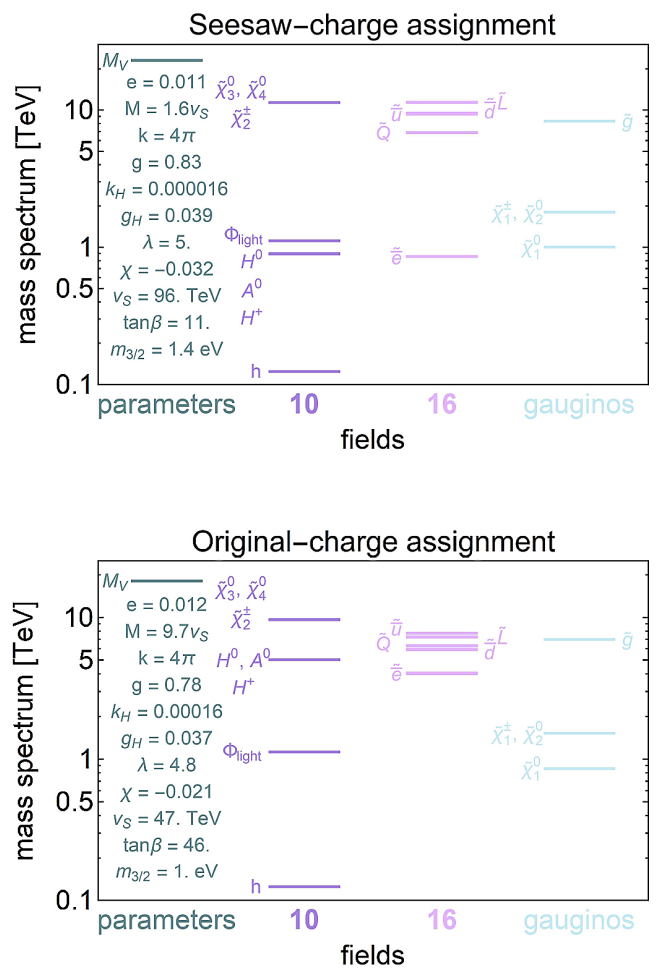

FIG. 6. Low-lying spectra for the seesaw-charge and originalcharge models with the lightest gravitino when $m_{h}$ is calculated at the 2-loop level.

$$
\begin{aligned}
\Delta m_{H_{u}}^{2}= & \frac{N_{C} y_{t}^{2}}{(4 \pi)^{2}}\left(m_{\tilde{t}_{R}}^{2} \ln \left(\frac{m_{\tilde{t}_{R}}^{2}}{\mu_{R}^{2}}\right)+m_{\tilde{t}_{L}}^{2} \ln \left(\frac{m_{\tilde{t}_{L}}^{2}}{\mu_{R}^{2}}\right)\right. \\
& \left.-m_{\tilde{t}_{R}}^{2}-m_{\tilde{t}_{L}}^{2}\right),
\end{aligned}
$$

where $N_{C}=3$ is the number of colors. At the renormalization scale, $\mu_{R}^{2}=m_{\tilde{t}_{R}} m_{\tilde{t}_{L}}$, this correction is negative as long as the stop masses are not too separated. This negative correction, in addition to the kinetic mixing $\chi$, enables EWSB in models with the seesaw-charge assignment.

Figure 5 shows why representative points in parameter space are excluded by the aforementioned constraints for the seesaw-charge and original-charge assignments when we calculate the Higgs mass at the 3-loop level. The most exclusive constraint is getting the Higgs mass correct. Points toward the bottom and left of these figures correspond to a Higgs which is too light. For the seesaw-charge assignment, the other MSSM Higgs scalars are often too light when the Higgs is too light. Points to the far right are excluded because $\delta \lambda_{h}$ from Eq. (15) is too large and the MSSM approximation breaks down.

The viable models with the lightest gravitinos are illustrated in Fig. 6. The equivalent figures with $m_{h}$ calculated at the 3-loop level are similar, with their spectra shifted slightly up.

SOFTSUSY requires $\mu$ to be 9.74 and $11.4 \mathrm{TeV}$ for the original- and seesaw-charge assignments, respectively, reducing the $\mu$ problem from the Planck scale to the $10-\mathrm{TeV}$ scale. One might be confused that the gauginos are near the sfermions. This is simply an artifact of maximizing the gaugino masses in units of $v_{S}$ before EWSB. The lightest gravitino masses for these 3-loop calculations are $1.9 \mathrm{eV}$ and $2.3 \mathrm{eV}$ for the original- and seesaw-charge assignments, respectively. Optimistically, we see that we can obtain gravitino masses as light as 1.0 and $1.4 \mathrm{eV}$ for the original- and seesaw-charge assignments when we calculate the Higgs at the 2-loop level. Even being conservative and calculating the Higgs mass at the 3-loop level still yields a sufficiently light gravitino to evade the current cosmological bound $m_{3 / 2}<4.7 \mathrm{eV}$. 


\section{CONCLUSION AND DISCUSSIONS}

We expand on the previous work of Hook and Murayama by finding models with lighter gravitinos, calculating EWSB, and enabling EWSB in models with the new, seesaw-charge assignment by introducing $U(1)$ kinetic mixing. Unlike typical, gauge-mediated models, we find that our vector-mediated models are cosmologically viable with light gravitinos. As can be extrapolated from Fig. 4, going to larger values of $e$ generally allows for lighter gravitinos. Since we use SOFTSUSY to approximate EWSB in our models, we only consider $e \lesssim 0.01$ to avoid invalidating this approximation. If we calculate EWSB without SOFTSUSY, we cannot consider values of $e$ much larger because the vacuum and thermal tunneling rates begin to matter. For values of $e \gtrsim 0.1$, we can no longer choose $k$ and $g$ to maximize the gaugino masses with respect to $v_{S}$. Satisfying the gaugino-mass bounds then increases $v_{S}$ which increases the gravitino mass. It is very interesting that the best we can do is not too far away from current cosmological limits. Near-future improvements in cosmological data, such as improvements in cosmic shear measurements at DES and Hyper Suprime-Cam, could completely rule out low-energy SUSY breaking.

\section{ACKNOWLEDGMENTS}

R. M. thanks Simon Knapen for many useful discussions and an introduction to SOFTSUSY. R. M. thanks Jason Evans and Marcin Badziak for helpful discussions. R. M. also thanks Katelin Schutz for comments on drafts. H. M. and R. M. thank T. T. Yanagida for discussions. R. M. is supported by an NSF graduate research fellowship. A. H. is supported by NSF Grant No. PHYS-1316699 and DOE Grant No. DE- SC0012012. This material is based upon work supported by the National Science Foundation Graduate Research Fellowship Program under Grant No. DGE 1106400. H. M. was supported by the U.S. DOE under Contract No. DE-AC02-05CH11231, and by the NSF under Grants No. PHY-1316783 and No. PHY1638509. H. M. was also supported by the JSPS Grant-inAid for Scientific Research (C) (No. 26400241 and No. 17K05409), MEXT Grant-in-Aid for Scientific Research on Innovative Areas (No. $15 \mathrm{H} 05887$ and No. 15K21733), and by WPI, MEXT, Japan. Any opinions, findings, and conclusions or recommendations expressed in this material are those of the author(s) and do not necessarily reflect the views of the National Science Foundation.
[1] H. Murayama, Supersymmetry phenomenology, in Proceedings of the Summer School in Particle Physics, Trieste, Italy, 1999 (World Scientific, Singapore, 2000), pp. 296-335.

[2] N. Cabibbo, G. R. Farrar, and L. Maiani, Massive photinos: Unstable and interesting, Phys. Lett. 105B, 155 (1981).

[3] L. Alvarez-Gaumé, M. Claudson, and M. B. Wise, Lowenergy supersymmetry, Nucl. Phys. B207, 96 (1982).

[4] M. Dine and W. Fischler, A phenomenological model of particle physics based on supersymmetry, Phys. Lett. 110B, 227 (1982).

[5] C. R. Nappi and B. A. Ovrut, Supersymmetric extension of the $\mathrm{SU}(3) \times \mathrm{SU}(2) \times \mathrm{U}(1)$ model, Phys. Lett. 113B, 175 (1982).

[6] T. Moroi, H. Murayama, and M. Yamaguchi, Cosmological constraints on the light stable gravitino, Phys. Lett. B 303, 289 (1993).

[7] A. de Gouvea, T. Moroi, and H. Murayama, Cosmology of supersymmetric models with low-energy gauge mediation, Phys. Rev. D 56, 1281 (1997).

[8] W. Buchmüller, P. Di Bari, and M. Plümacher, Some aspects of thermal leptogenesis, New J. Phys. 6, 105 (2004).

[9] K. Osato, T. Sekiguchi, M. Shirasaki, A. Kamada, and N. Yoshida, Cosmological constraint on the light gravitino mass from CMBlensing and cosmic shear, J. Cosmol. Astropart. Phys. 06 (2016) 004.
[10] M. Viel, J. Lesgourgues, M. G. Haehnelt, S. Matarrese, and A. Riotto, Constraining warm dark matter candidates including sterile neutrinos and light gravitinos with WMAP and the Lyman-alpha forest, Phys. Rev. D 71, 063534 (2005).

[11] M. Kawasaki, K. Kohri, T. Moroi, and A. Yotsuyanagi, Big-bang nucleosynthesis and gravitino, Phys. Rev. D 78, 065011 (2008).

[12] M. Kawasaki, K. Kohri, T. Moroi, and Y. Takaesu, Revisiting big-bang nucleosynthesis constraints on long-lived decaying particles, Phys. Rev. D 97, 023502 (2018).

[13] M. A. Ajaib, I. Gogoladze, F. Nasir, and Q. Shafi, Revisiting mGMSB in light of a $125 \mathrm{GeV}$ Higgs, Phys. Lett. B 713, 462 (2012).

[14] P. Draper, P. Meade, M. Reece, and D. Shih, Implications of a $125 \mathrm{GeV}$ Higgs for the MSSM and low-scale SUSY breaking, Phys. Rev. D 85, 095007 (2012).

[15] P. Batra, A. Delgado, D. E. Kaplan, and T. M. P. Tait, The Higgs mass bound in gauge extensions of the minimal supersymmetric standard model, J. High Energy Phys. 02 (2004) 043.

[16] A. Maloney, A. Pierce, and J. G. Wacker, D-terms, unification, and the Higgs mass, J. High Energy Phys. 06 (2006) 034.

[17] T. T. Yanagida, N. Yokozaki, and K. Yonekura, Higgs boson mass in low scale gauge mediation models, J. High Energy Phys. 10 (2012) 017. 
[18] X. Lu, H. Murayama, J. T. Ruderman, and K. Tobioka, A Natural Higgs Mass in Supersymmetry from Nondecoupling Effects, Phys. Rev. Lett. 112, 191803 (2014).

[19] N. Craig, S. Knapen, D. Shih, and Y. Zhao, A complete model of low-scale gauge mediation, J. High Energy Phys. 03 (2013) 154.

[20] T. T. Yanagida and K. Yonekura, A conformal gauge mediation and dark matter with only one parameter, Phys. Lett. B 693, 281 (2010).

[21] J. J. Heckman, P. Kumar, C. Vafa, and B. Wecht, Electroweak symmetry breaking in the DSSM, J. High Energy Phys. 01 (2012) 156.

[22] J. L. Evans, M. Ibe, and T. T. Yanagida, The lightest Higgs boson mass in the MSSM with strongly interacting spectators, Phys. Rev. D 86, 015017 (2012).

[23] R. Kitano, M. A. Luty, and Y. Nakai, Partially composite Higgs in supersymmetry, J. High Energy Phys. 08 (2012) 111.

[24] A. Hook and H. Murayama, Low-energy Supersymmetry breaking without the gravitino problem, Phys. Rev. D 92 , 015004 (2015).

[25] S. Davidson, E. Nardi, and Y. Nir, Leptogenesis, Phys. Rep. 466, 105 (2008).

[26] J. L. Evans, N. Nagata, and K. A. Olive, SU(5) grand unification in pure gravity mediation, Phys. Rev. D 91, 055027 (2015).

[27] F. Brummer, A. Hebecker, and M. Trapletti, SUSY breaking mediation by throat fields, Nucl. Phys. B755, 186 (2006).

[28] L. Randall and R. Sundrum, Out of this world supersymmetry breaking, Nucl. Phys. B557, 79 (1999).

[29] G. F. Giudice, M. A. Luty, H. Murayama, and R. Rattazzi, Gaugino mass without singlets, J. High Energy Phys. 12 (1998) 027.

[30] L. Girardello and M. T. Grisaru, Soft breaking of supersymmetry, Nucl. Phys. B194, 65 (1982).

[31] S. Ferrara, L. Girardello, and F. Palumbo, A general mass formula in broken supersymmetry, Phys. Rev. D 20, 403 (1979).

[32] L. J. Hall and I. Hinchliffe, Simple viable models of lowenergy supersymmetry, Phys. Lett. 112B, 351 (1982).

[33] M. Nardecchia, A. Romanino, and R. Ziegler, Tree level gauge mediation, J. High Energy Phys. 11 (2009) 112.

[34] M. Monaco, M. Nardecchia, A. Romanino, and R. Ziegler, Extended tree-level gauge mediation, J. High Energy Phys. 10 (2011) 022.

[35] G. Arcadi, L. Di Luzio, and M. Nardecchia, Gravitino dark matter in tree level gauge mediation with and without $R$-parity, J. High Energy Phys. 12 (2011) 040.

[36] K.-I. Izawa and T. Yanagida, Dynamical supersymmetry breaking in vectorlike gauge theories, Prog. Theor. Phys. 95, 829 (1996).

[37] K. A. Intriligator and S. D. Thomas, Dynamical supersymmetry breaking on quantum moduli spaces, Nucl. Phys. B473, 121 (1996).

[38] K. R. Dienes, C. F. Kolda, and J. March-Russell, Kinetic mixing and the supersymmetric gauge hierarchy, Nucl. Phys. B492, 104 (1997).
[39] S. R. Coleman, The fate of the false vacuum. 1. Semiclassical theory, Phys. Rev. D 15, 2929 (1977).

[40] F. C. Adams, General solutions for tunneling of scalar fields with quartic potentials, Phys. Rev. D 48, 2800 (1993).

[41] S. Coleman, Aspects of Symmetry: Selected Erice Lectures (Cambridge University Press, Cambridge, England, 1985), p. 008 .

[42] I. Yu. Kobzarev, L. B. Okun, and M. B. Voloshin, Bubbles in metastable vacuum, Sov. J. Nucl. Phys. 20, 644 (1975).

[43] E. Poppitz and S.P. Trivedi, Some remarks on gauge mediated supersymmetry breaking, Phys. Lett. B 401, 38 (1997).

[44] B. C. Allanach, SOFTSUSY: A program for calculating supersymmetric spectra, Comput. Phys. Commun. 143, 305 (2002).

[45] B. C. Allanach, A. Bednyakov, and R. R. de Austri, Higher order corrections and unification in the minimal supersymmetric standard model: SOFTSUSY3.5, Comput. Phys. Commun. 189, 192 (2015).

[46] R. V. Harlander, J. Klappert, and A. Voigt, Higgs mass prediction in the MSSM at three-loop level in a pure $\overline{\mathrm{DR}}$ context, Eur. Phys. J. C 77, 814 (2017).

[47] P. Kant, R. V. Harlander, L. Mihaila, and M. Steinhauser, Light MSSM Higgs boson mass to three-loop accuracy, J. High Energy Phys. 08 (2010) 104.

[48] ATLAS Collaboration, Search for squarks and gluinos in final states with jets and missing transverse momentum using $36 \mathrm{fb}^{-1}$ of $\sqrt{s}=13 \mathrm{TeV} p p$ collision data with the ATLAS detector, CERN, Geneva, Technical Report No. ATLAS-CONF-2017-022, 2017.

[49] A. M. Sirunyan et al. (CMS Collaboration), Search for supersymmetry in Multijet events with missing transverse momentum in proton-proton collisions at $13 \mathrm{TeV}$, Phys. Rev. D 96, 032003 (2017).

[50] G. Aad et al. (ATLAS Collaboration), Search for photonic signatures of gauge-mediated supersymmetry in $8 \mathrm{TeV} p p$ collisions with the ATLAS detector, Phys. Rev. D 92, 072001 (2015).

[51] C. Patrignani et al. (Particle Data Group), Review of particle physics, Chin. J. Phys. C 40, 100001 (2016).

[52] G. Degrassi, S. Di Vita, J. Elias-Miro, J. R. Espinosa, G. F. Giudice, G. Isidori, and A. Strumia, Higgs mass and vacuum stability in the Standard Model at NNLO, J. High Energy Phys. 08 (2012) 098.

[53] M. Aaboud et al. (ATLAS Collaboration), Measurement of the top quark mass in the $t \bar{t} \rightarrow$ dilepton channel from $\sqrt{\mathrm{s}}=$ $8 \mathrm{TeV}$ ATLAS data, Phys. Lett. B 761, 350 (2016).

[54] N. Craig, F. D'Eramo, P. Draper, S. Thomas, and H. Zhang, The hunt for the rest of the Higgs bosons, J. High Energy Phys. 06 (2015) 137.

[55] M. Aaboud et al. (ATLAS Collaboration), Search for additional heavy neutral Higgs and gauge bosons in the ditau final state produced in $36 \mathrm{fb}^{-1}$ of $p p$ collisions at $\sqrt{s}=13 \mathrm{TeV}$ with the ATLAS detector, J. High Energy Phys. 01 (2018) 055. 